\title{
Therapeutic Benefit of Addition of Cetirizine to Ambroxol in Fixed Dose Combination in the Treatment of Productive Cough in Children: A Prospective, Multi-center Study
}

\author{
Amit Garg ${ }^{1}$, * Bharath Venkata Ramisetty ${ }^{2}$, Nirmal Choraria ${ }^{3}$, Kirti Sisodiya ${ }^{4}$, \\ Monidipa Banerjee ${ }^{5}$, Shyam Akku' \\ ${ }^{1}$ Global Medical Affairs, Dr. Reddy's Laboratories, Banjara Hills, Hyderabad, India \\ ${ }^{2}$ Narayana Medical College and Hospital, Opposite Narayana Engineering College, Chinthareddypalem, Nellore, India \\ ${ }^{3}$ Nirmal Hospital, Centre Point, Near Kadiwala School, Surat, India \\ ${ }^{4}$ BAPS Hospital, Shri Pramukh Swami Maharaj Marg, Adajan Char Rasta, Adajan, Surat, India \\ ${ }^{5}$ Peerless Hospital \& 'B.K. Roy Research Center360 Panchasayar, Kolkata, India \\ Email address: \\ amitgarg.pharm@gmail.com (A. Garg) \\ ${ }^{*}$ Corresponding author
}

To cite this article:

Amit Garg, Bharath Venkata Ramisetty, Nirmal Choraria, Kirti Sisodiya, Monidipa Banerjee, Shyam Akku. Therapeutic Benefit of Addition of Cetirizine to Ambroxol in Fixed Dose Combination in the Treatment of Productive Cough in Children: A Prospective, Multi-center Study. American Journal of Internal Medicine. Vol. 5, No. 6, 2017, pp. 129-135. doi: 10.11648/j.ajim.20170506.14

Received: January 28, 2017; Accepted: February 14, 2017; Published: November 28, 2017

\begin{abstract}
Productive cough is a common problem in children and is accompanied by disruption of sleep and routine daily activities leading to a deterioration in quality of life. We evaluated the efficacy, safety and tolerability of cetirizine and ambroxol fixed dose combination (FDC) as compared with ambroxol in these patients. We conducted open-label, prospective, multicenter study attending outpatient department with cough $\geq 12$ hours; were screened to receive either cetirizine and ambroxol FDC (AC group) or ambroxol alone (AX group). Primary efficacy variables were evaluation of total symptom score (TSS) for cough and secondary efficacy variables included evaluation of total nasal symptom score (TNSS); assessment of cough frequency; number of awakenings due to cough; and time to complete relief. Out of 250 children included, 246 completed the study with a statistically significant improvement in TSS from baseline to day $3(p=0.029)$ and day $7(p=0.048)$ in the $\mathrm{AC}$ group as compared with AX group. Improvement in TNSS was better in the AC group from baseline to day 3 $(p<0.0001)$ and day $7(p=0.016)$ as compared with the AX group. Greater proportion of children in the AC group recovered completely from cough by day 7 (97.67\%) as compared with the AX group (78.63\%). As side effects, only two children of the AC group experienced mild AEs (drowsiness). We thus conclude that FDC demonstrated improved efficacy and safety and was well tolerated as compared with ambroxol alone in children with productive cough.
\end{abstract}

Keywords: Cetirizine, Ambroxol, Productive Cough, Children, Total Symptom Score

\section{Introduction}

Cough may be considered as a protective reflex especially when the secretions are copious [1]. Productive cough is often characterized by the presence of mucoid or mucopurulent secretions due to irritants [2]. Productive cough is a natural reflex which helps clear the airways of the excessive secretions in addition to preventing the entry of foreign matter in the airways [3]. A study evaluating the prevalence of productive cough in children below 13 years of age reported productive cough in $7.2 \%$ of the study population ( $\mathrm{n}=173)$ [4]. Most children experience cough as a result of acute viral infections and a very small proportion of patients experience cough as a result of asthma or other causes. Additionally, it is important to mention that most of coughs observed in children are due to undifferentiated acute 
respiratory infections [5]. In some cases, the cough may not be infective, and persistent however; chesty sounding cough which lasts for more than four weeks can be considered to occur as a result of chest infections [6]. Irritant factors that contribute to the occurrence of cough include environmental factors viz. irritant gases; exposure to tobacco smoke; indoor pollution; and dampness at home [7]. Treatment of productive cough is extremely important and investigation of underlying lung or other disease is recommended in children with cough for more than 4 weeks [8].

Ambroxol, a mucokinetic agent [9] shows secretolytic, anti-inflammatory, and local anesthetic effect and has been shown to reduce the symptoms of cough [10]. Cetirizine selectively inhibits peripheral histamine (H1) receptors and has anti-allergic properties, there by effectively reducing cough intensity and frequency in patients with allergic cough [11]. A study conducted to evaluate the effect of antihistaminic agents in children showed that the administration of cetirizine improves cough due to allergy in children [12]. The pharmacodynamic properties of both cetirizine and ambroxol could be used for the symptomatic relief of productive cough associated with allergic rhinitis when both antihistamine and mucokinetic agents are desired.

The symptoms of cough are different in children as compared with adults. [13]. Cough is accompanied by disruption of sleep and routine daily activities of the child leading to a deterioration in quality of life (QoL) [7, 14]. These factors necessitate optimal treatment of cough in children. The combination of cetirizine and ambroxol was hypothesized to provide effective symptom relief in children with productive cough. Presently, there is no evidence available on the effectiveness of this FDC in the treatment of productive cough. Prescription of an FDC is associated with better patient compliance and is considered to be more costeffective than two individual medications [15]. This study was conducted to compare the efficacy, safety and tolerability of FDC of cetirizine and ambroxol with ambroxol alone in the treatment of productive cough in children.

\section{Materials and Methods}

\subsection{Study Characteristics}

This was an open-label, prospective, multicenter study conducted between June 2015 and October 2015. The study duration was for a maximum of 7 days for each patient at the discretion of the physician. The study was conducted across four different sites in India (two from Surat; one each at Nellore and Kolkata).

The study was conducted in accordance to the Declaration of Helsinki, and Good Clinical Practice (GCP). The study protocol and any amendments to the same were approved by the Institutional Review Board (IRB) and the Independent Ethic Committee (IEC) of the respective study centers. A written informed consent was obtained from the parents/guardians of all children prior to the initiation of the study. Patient anonymity was preserved. The study is registered at Clinical Trials Registry
- India (TRI/2015/09/006198).

\subsection{Inclusion and Exclusion Criteria}

Children attending the outpatient department with a complaint of cough lasting for more than 12 hours were screened. Children of either gender between 6 and 18 years of age; with productive cough (cough with expectoration) associated with any of the nasal symptoms for at least 8 hours prior to study initiation were included. Additionally, children willing to provide assent and parents/guardian willing to provide written informed consent were included.

Children using any throat lozenge, throat spray, cough drop, menthol-containing product, or any product with demulcent properties, any cold medication viz. nasal or systemic decongestants, antihistaminic agents, expectorants, or antitussives within last 8 hours of study initiation were excluded from the study. Those children with severe cough requiring hospitalization, active pulmonary diseases such as bronchopneumonia, bronchial asthma or tuberculosis, a known allergy to ambroxol and/or cetirizine, fever within 8 hours of study initiation, any form of infection, or allergic rhinitis were excluded. Additionally, patients with cough for longer duration of time were excluded from the study.

\subsection{Treatment Allocation}

Patients received either Relent plus Syrup (manufacturer: Dr. Reddy's Laboratories, India) containing cetirizine hydrochloride $5 \mathrm{mg}$ and ambroxol hydrochloride $30 \mathrm{mg}$ per 5 $\mathrm{mL} /$ any pharmacological equivalent in the market (AC group) or Mucolite Syrup (manufacturer: Dr. Reddy's Laboratories, India) containing ambroxol hydrochloride 30 mg per $5 \mathrm{~mL} /$ any pharmacological equivalent in the market (AX group), given twice daily. Both the physician and the patient were aware of the treatments being received by the patients.

\subsection{Study Assessments}

\subsubsection{Efficacy Variables}

The primary efficacy variables of the study were to evaluate the TSS for cough calculated by summing individual scores of four symptoms including sputum volume, sputum viscosity, difficulty of expectoration and the severity of cough; and improvements in TSS scores from baseline (day 0 ) to end of study period was evaluated [16].

The secondary efficacy variables of the study included evaluation of the TNSS for cough calculated by summing by four symptoms including nasal discharge, nasal stuffiness, nasal itching, and sneezing. Additionally, the improvement in TNSS scores from baseline (day 0) to end of study period were calculated [17]. Both TSS and TNSS were 4-point scale in which a value of 0 " signified absence of symptoms (no signs or symptoms); 1 signified presence of mild symptoms (sign/symptom clearly present, but minimal awareness; easily tolerated); 2 signifies the presence of moderate symptoms (definite awareness of sign/symptom that is bothersome but 
tolerable); and " 3 " signified the presence of severe symptoms (signs and symptoms that are hard to tolerate, and cause disturbance in daily activities and/or sleep).

The other secondary efficacy variables included assessment of cough frequency (evaluated daily); number of awakenings due to cough (evaluated on a daily basis and overall improvement during the study); and time to complete relief (number of days taken for complete relief i.e., for the symptom score to become 0 for all symptoms including sputum volume, sputum viscosity, difficulty of expectoration, and severity of cough).

\subsubsection{Safety and Tolerability Variables}

All AEs reported by the children were documented during the study period. The presence of drowsiness/sleepiness; and concentration impairment (patients report whether there is concentration during studies or reading is impaired) during the day were evaluated. Patient compliance with the study medication was recorded during the study and at follow-up visits. Tolerability of the study medication was evaluated with the GATT scale. The GATT was a 5-point scale in which the value of " 1 " signified excellent tolerability and the value of " 5 " signified worst tolerability. No specific laboratory assessments were done during the study as it was anticipated that the study medication may not cause any laboratory abnormalities [18].

\subsubsection{Patient Follow-up}

The study comprised of four visits viz. visit 1 or screening (day -2 through day 0); visit 2 or enrolment and baseline data (day 0 ); visit 3 or follow-up (day $3 \pm 1$ ); and visit 4 or study completion (day $7 \pm 1$ ). On visit 1 , the patients were screened for eligibility in the study and underwent allocation and study assessments (day 0). Treatment decision and prescription of medication was done on day 0. During the follow-up phase through study completion, study assessments and evaluation of compliance were done.

\subsection{Statistical Analysis}

To reject the null hypothesis, or if there is truly no difference between the AC group (study) and AX group, 174 patients were required with $95 \%$ confidence interval. Considering the age of patients and the declined season of cough, we anticipate a huge drop-out rate. Thus, for acquiring complete data for 174 patients, 250 patients were included in the study. Descriptive statistics, unpaired' $t$ ' test and Mann-Whitney ' $U$ ' test were used for analysis. A $p$ value of less than 0.05 was considered statistically significant.

\section{Results}

\subsection{Study Patients}

Overall, 250 children were included in the study of which 246 children completed the study. The mean age of the children of the AC group was $8.78 \pm 2.38$ years and that of the AX group was $9.11 \pm 2.18$ years. Majority of the children in both groups were boys (AC group: 57.36\%; AX group:
59.83\%). The AC group included 129 children and the AX group included 117 children. The detailed demographic data is presented in Table 1.

Table 1. Demographic Details of the Patients.

\begin{tabular}{|c|c|c|c|}
\hline & $\begin{array}{l}\text { Ambroxol + } \\
\text { Cetirizine }(N=129)\end{array}$ & $\begin{array}{l}\text { Ambroxol } \\
(\mathrm{N}=117)\end{array}$ & $\begin{array}{l}\text { - } \\
\text { value }\end{array}$ \\
\hline \multicolumn{4}{|l|}{ Age (yr) } \\
\hline Mean \pm SD & $8.78 \pm 2.38$ & $9.11 \pm 2.18$ & 0.259 \\
\hline Median & 8.11 & 9.00 & \\
\hline Range (min-max) & $9(6-15)$ & $9.06(6-15.06)$ & \\
\hline \multicolumn{4}{|l|}{ Height (cm) } \\
\hline Mean \pm SD & $126.85 \pm 16.81$ & $130.43 \pm 15.19$ & 0.082 \\
\hline Median & 125.9 & 129.5 & \\
\hline Range (min-max) & $93.4(66-159.4)$ & $65(100-165)$ & \\
\hline \multicolumn{4}{|l|}{ Weight (Kg) } \\
\hline Mean \pm SD & $30.21 \pm 9.00$ & $31.9 \pm 10.65$ & 0.159 \\
\hline Median & 29.6 & 29 & \\
\hline Range (min-max) & $43.6(14.8-58.4)$ & $49.2(16.2-65.4)$ & \\
\hline \multicolumn{4}{|l|}{ BMI $\left(\mathrm{Kg} / \mathrm{m}^{2}\right)$} \\
\hline Mean \pm SD & $18.51 \pm 4.78$ & $18.56 \pm 3.94$ & 0.928 \\
\hline Median & 17.21 & 17.37 & \\
\hline Range (min-max) & $35.2(0.0-35.2)$ & $20.05(8.8-28.9)$ & \\
\hline \multicolumn{4}{|l|}{ Gender, n (\%) } \\
\hline Boy & $74(57.36)$ & $70(59.83)$ & 0.695 \\
\hline \multicolumn{4}{|l|}{$\begin{array}{l}\text { Subjects having } \\
\text { symptoms }\end{array}$} \\
\hline $\begin{array}{l}\text { Cough bouts/ episodes } n \\
(\%)\end{array}$ & $129(100)$ & $117(100)$ & 1.000 \\
\hline Mean \pm SD & $14.5 \pm 9.1$ & $14.1 \pm 7.1$ & \\
\hline Median & 12.0 & 12.0 & \\
\hline Range (min-max) & $47(3-50)$ & $28(2-30)$ & \\
\hline Night awakenings n (\%) & $123(95.35)$ & $114(97.44)$ & 0.5946 \\
\hline Mean \pm SD & $3.52 \pm 2.32$ & $3.90 \pm 2.01$ & \\
\hline Median & 3.0 & 4.0 & \\
\hline Range (min-max) & $15(0-15)$ & $10(0-10)$ & \\
\hline Standard Deviation: SD; & 3ody Mass Index: BM & & \\
\hline
\end{tabular}

\subsection{Primary Efficacy Variable}

The primary end point was Total Symptom Score (TSS) for Cough. The mean baseline TSS values for cough were well matched between the two groups (AC: 11.59 \pm 1.82 ; AX: $11.41 \pm 2.13)$. The change from baseline in the mean TSS scores in AC group was 3.77 and 7.51 on day 3 and day 7 , similarly for AX groups it was 3.37 and 6.92, respectively. The improvement in TSS from baseline to day 3 and day 7 was significantly more in children of the $\mathrm{AC}$ group as compared with those of the AX group, $p=0.029$ and $p=0.048$ respectively (Figure 1A).

\subsection{Secondary Efficacy Variables}

\subsubsection{Total Nasal Symptom Score (TNSS) for Cough}

The mean baseline TNSS values for cough were well matched between the two groups (AC: 9.76 \pm 1.95 ; AX: $9.65 \pm 1.94)$. The improvement in TNSS from baseline to day $3(p<0.0001)$ and day $7(p=0.016)$ was significantly more in children of the AC group as compared with those of the AX group (Figure 1B). 


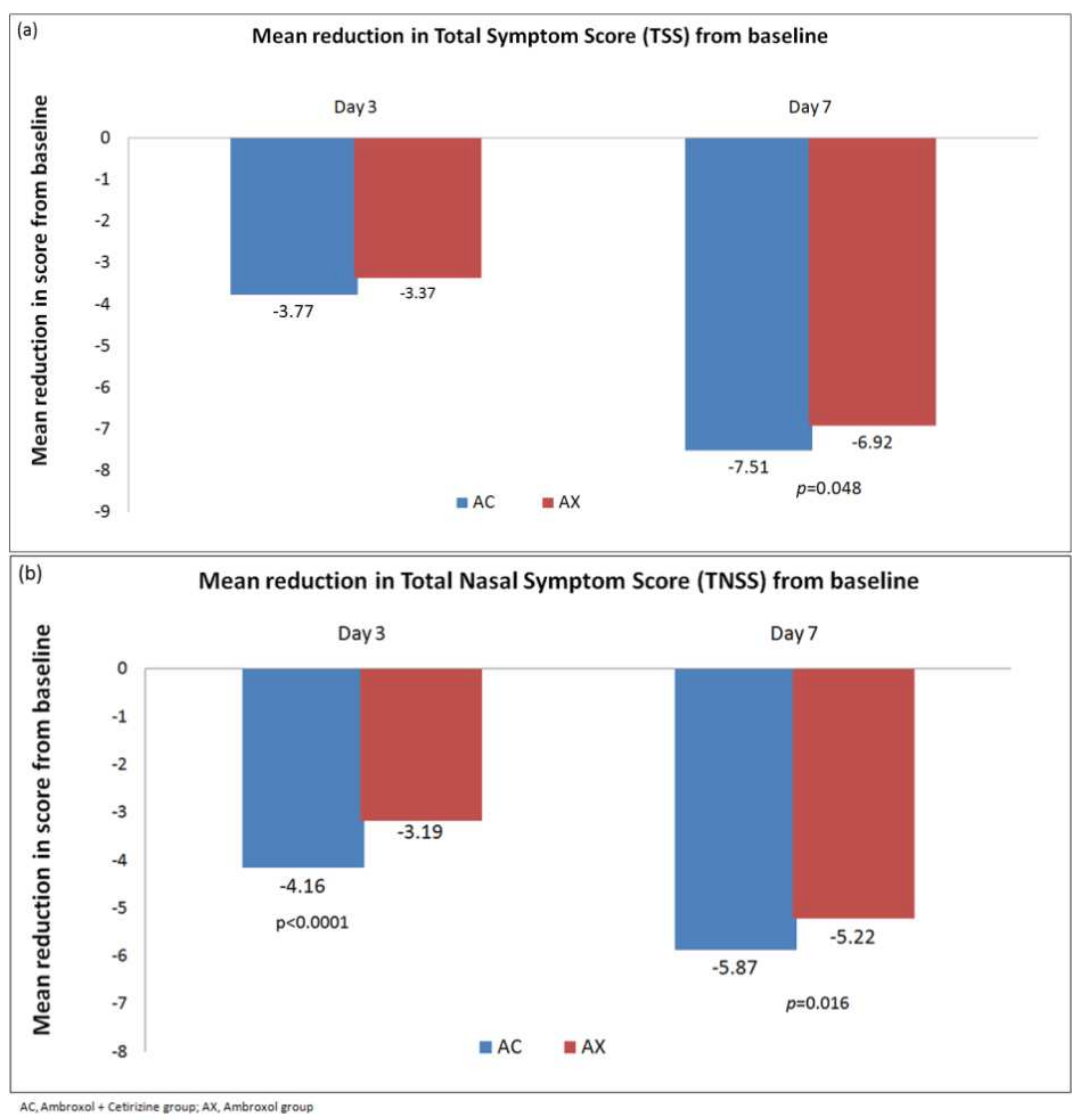

Figure 1. (A) Mean Reduction in TSS and (B) TNSS from Baseline to Day 3 and Day 7.

\subsubsection{Improvement in Cough and Related Efficacy Variables}

Cough Frequency: The reduction in cough frequency was similar at day 3 and day 7 in both AC and AX groups (Table 2). A greater proportion of children in the $\mathrm{AC}$ group recovered completely from cough on day $3(8.53 \%)$ and day $7(71.32 \%)$ as compared with the children of the AX group (day 3: 2.56\%; day 7: 64.96\%). However; the difference between the two groups was not statistically significant at both day 3 ( $p=0.0813)$ and day $7(p=0.3506)$.

Number of Awakenings: The proportion of children who completely stopped awakening at night and the median number of night awakening was similar between the two groups on day 7 (Table 2).

Table 2. Changes in Cough Frequency and Number of Awakenings during the Study Period.

\begin{tabular}{llll}
\hline & Ambroxol + Cetirizine & Ambroxol & \\
\hline Cough frequency & Median (Range) & Median (Range) & $p$ \\
Baseline & $3(1-3)$ & $3(1-3)$ & 0.803 \\
Day 3 & $2(0-2)$ & $2(0-2)$ & 0.069 \\
Day 7 & $1(0-2)$ & $1(0-1)$ & 0.206 \\
Night awakenings & & & 0.056 \\
Baseline & $3(0-15)$ & $4(0-10)$ & 0.010 \\
Day 3 & $1(0-5)$ & $1(0-9)$ & 0.304 \\
Day 7 & $0(0-1)$ & $0(0-2)$ & $p$ \\
Patients not having cough & $n(\%)$ & $n(\%)$ & - \\
Baseline & $0(-)$ & $0(-)$ & 0.0813 \\
Day 3 & $11(8.53)$ & $3(2.56)$ & 0.3506 \\
Day 7 & $92(71.32)$ & $76(64.96)$ & \\
Patients not having night awakenings & & & \\
Baseline & $6(4.65)$ & $3(2.56)$ & 0.5946 \\
Day 3 & $51(39.53)$ & $38(32.48)$ & 0.3094 \\
Day 7 & $116(89.92)$ & $112(95.73)$ & 0.133 \\
\hline
\end{tabular}

Time to Complete Relief: In AC group 10 (7.75\%) children got complete relief by day 3 and $126(97.67 \%)$ by day 7 . Only 3 children didn't have complete relief by day 7 . In AX group, $4(3.42 \%)$ children got complete relief by day 3 and $92(78.63 \%)$ by day 7 while $25(21.37 \%)$ children did not complete relief by day 7 . Significantly higher proportion of 
children of the AC group got complete relief as compared to AX group by day $7(p<0.0001)$. (Figure 2$)$. More number of patients in the AC group got complete relief earlier (by day 5), as compared to AX group.

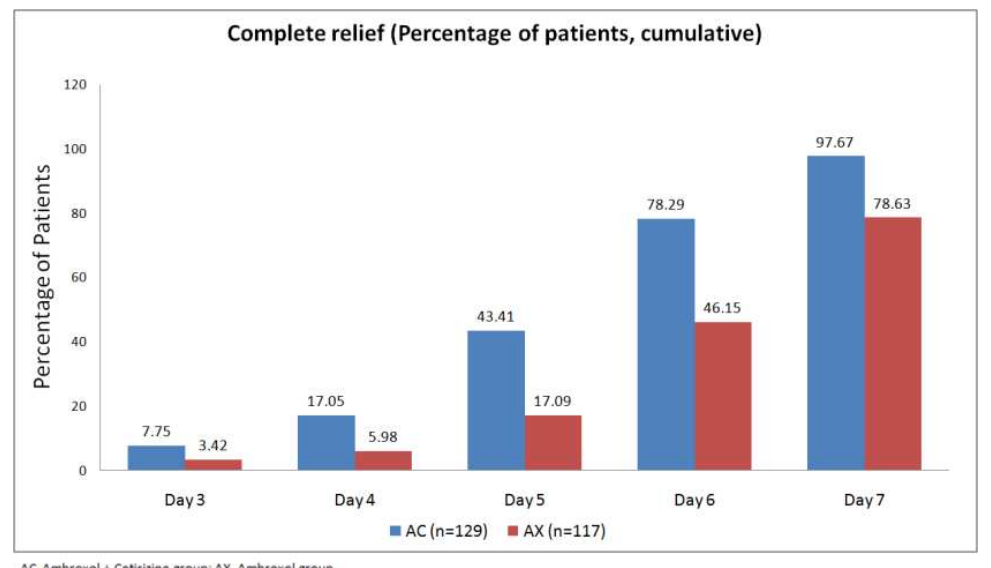

Figure 2. Time to Complete Relief from Cough.

\subsection{Safety and Tolerability Variables}

Vital parameters viz. pulse rate, respiration rate, systolic and diastolic blood pressure, and body temperature showed no changes during the study period. Of the 246 children who completed the study, only two children $(0.8 \%)$ experienced AEs and both were from the $\mathrm{AC}$ group. Both children experienced drowsiness which was mild in severity. None of the children in the AX group reported AEs during the study period.

Concentration impairment was not reported by any of the children who participated in the study. All children adhered to the therapy with $100 \%$ compliance in the $\mathrm{AC}$ group throughout the study. In the AX group, not all but most of the children (99.12\%) were compliant to the therapy on day 7 .

\subsection{Global Assessment of Tolerability to Therapy (GATT)}

The GATT was assessed by physicians and by parents/guardians for children of both the groups. Poor tolerability was observed in negligible proportion of children in the AX group (0.85\%) and no children in the $\mathrm{AC}$ group. Although a greater proportion of children in the $\mathrm{AC}$ group (by physician: $82.95 \%$; by parent/guardian: $80.62 \%$ ) exhibited excellent tolerability, the difference between the two groups did not achieve statistical significance (Figure 3).

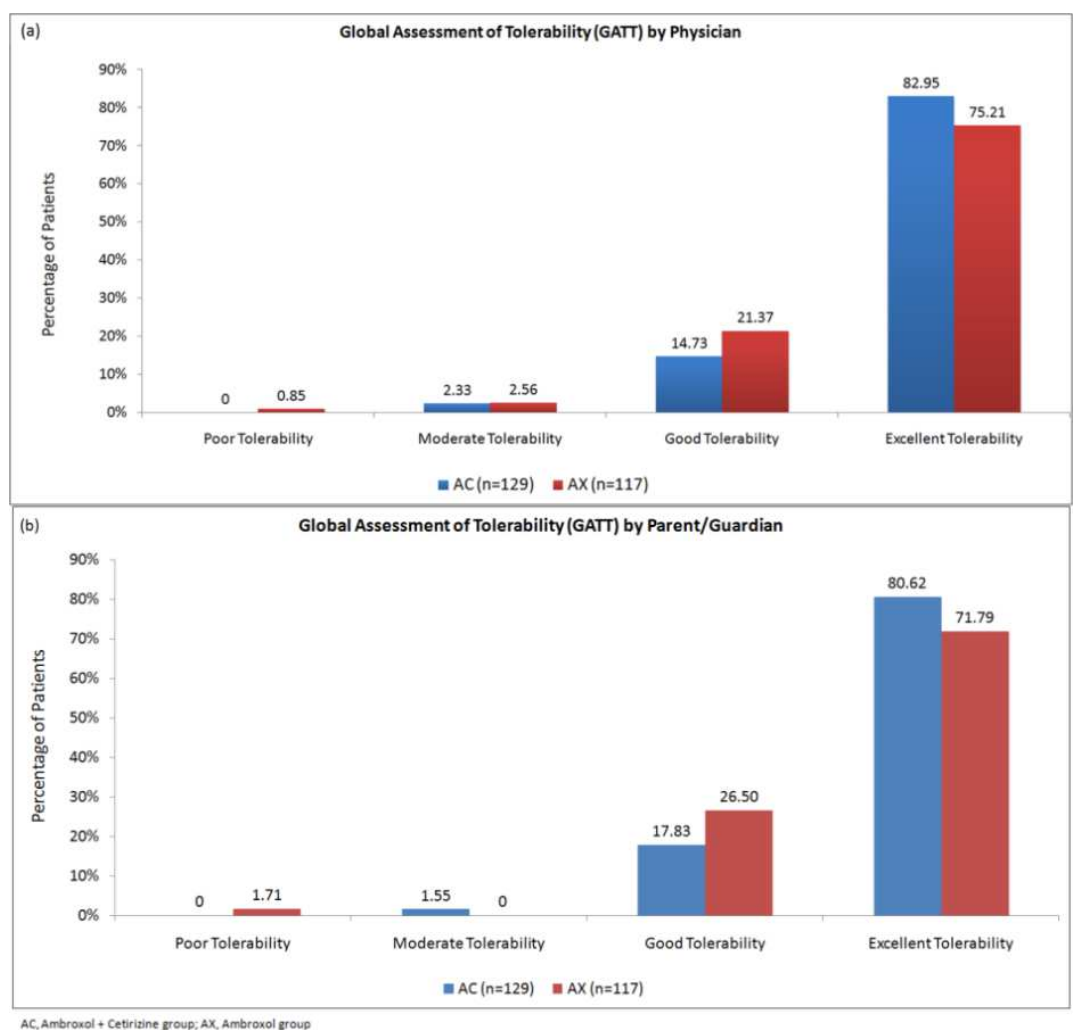

Figure 3. Tolerability of Treatments as Assessed with Global Assessment of Tolerability (GATT) Scale. 


\section{Discussion}

This study compared the efficacy of cetirizine and ambroxol FDC with ambroxol through change in TSS and TNSS scores and additionally assessed the change in cough frequency, number of awakenings at night due to cough, and the time duration taken for complete cough relief. Improvement in TSS and TNSS in the AC group was significant as compared with the ambroxol group. Proportion of children with improvement in cough was considerably high in children who received cetirizine ambroxol FDC as compared with children who had received only ambroxol. The study demonstrated that the number of night awakenings reduced in both groups, but the proportion of children with the improvement was higher in the $\mathrm{AC}$ group as compared with the AX group. More number of patients had complete relief from cough rapidly in the AC group as compared with the AX groups and complete relief was observed in almost all of the children in the AC group as compared to only three fourth of the children in the AX group by day 7 of the study. The improved outcomes in children of the AC group have shown that the cetirizine and ambroxol FDC is favorable in children with productive cough.

The study included children between 6 and 18 years of age. The study considered inclusion of children mainly because cough is said to be more prevalent in children than in adults. This age group was considered for analysis in the present study due to the different symptom pattern of cough in children and associated discomforts of cough viz. disruption of sleep [7]. The most common causes of cough in children are upper or lower respiratory tract infections, asthma, or gastroesophageal reflux disease (GERD). The less common causes include the aspiration of foreign bodies, post-infectious cough, or pertussis [19]. A review conducted by Chung et al reported that the presence of environmental pollutants is one of the most common causes for cough in children particularly in school going children [20]. The British Thoracic Society (BTS) recommends the use of antihistamines in children with productive cough and in allergic cough that has occurred in the pollen season [21]. While cetirizine is essential to combat allergic cough, inclusion of ambroxol to the treatment regimen is essential as it promotes cough clearance of mucous and facilitates reduction of surface adhesion between mucous and airway epithelium [9].

Occurrence of productive cough in children requires adequate management for better relief and uninterrupted sleep [22]. Combination of an anti-histamine (cetirizine) and a mucokinetic (ambroxol) was hypothesized to be more effective as compared with the mucokinetic alone. It is a known fact that most of the medications available for the treatment of cough and cold include decongestants, cough suppressants, anti-histaminic agents, and expectorants [23] and the administration of multiple medication combinations is often associated with drug overdose and unintended adverse events [24]. Keeping the safety aspect also into consideration for the age group included in this study, the cetirizine and ambroxol combination was investigated. The study results have demonstrated that in addition to the improvement in productive cough, children tolerated the combination well and there were no serious adverse events reported. Only two children reported the presence of drowsiness with the combination therapy which was mild.

Additional benefits of using an FDC in the treatment of cough in children is treatment compliance. Administration of a single medication with a combination is easier among this age group than administration of more than one medication. These benefits have led to an increase in the availability of FDCs for the treatment of cough and cold. Over the past decade, the availability of liquid FDCs for the treatment of cough has increased from $67.9 \%$ in 2004 to $71.8 \%$ in 2007 [25].

Open label study design may be considered as the main limitations of the present study. Assessment of the efficacy and tolerability of ambroxol and cetirizine FDC would have been clearer with a blinded study design. Although there are guidelines for the assessment and management of cough in children, there is a need to consider FDC in these considering the age group which can ensure safer clinical practice and adequate treatment of children.

\section{Conclusions}

The use of cetirizine and ambroxol as fixed dose combination that is approved by regulators is effective in the management of productive cough in children. This combination effectively reduces the overall symptoms, number of nigh awakenings, improves cough frequency in children ensuring better complete relief and was well tolerated in this population.

\section{List of Abbreviations}

$\begin{array}{lll}1 & \text { FDC } & \text { Fixed Dose Combination } \\ 2 & \text { GCP } & \text { Good Clinical Practice } \\ 3 & \text { IRB } & \text { Institutional Review Board } \\ 4 & \text { IEC } & \text { Independent Ethic Committee } \\ 5 & \text { AC } & \text { Ambroxol + Cetirizine } \\ 6 & \text { AX } & \text { Ambroxol } \\ 7 & \text { TSS } & \text { Total Symptom Score } \\ 8 & \text { TNSS } & \text { Total Nasal Symptom Score } \\ 9 & \text { AE } & \text { Adverse Event } \\ 10 & \text { SAE } & \text { Serious Adverse Event } \\ 11 & \text { GATT } & \text { Global Assessment of Tolerability to Therapy } \\ 12 & \text { SD } & \text { Standard Deviation } \\ 14 & \text { GERD } & \text { Gastroesophageal Reflux Disease } \\ 15 & \text { BTS } & \text { British Thoracic Society }\end{array}$

\section{Acknowledgements}

Financial support for medical editorial assistance was provided by Dr. Reddy's Laboratories Ltd. 


\section{References}

[1] World Health Organization. Cough and Cold Remedies for the Treatment of Acute Respiratory Infections in Young Children. 2001. Available from:

http://apps.who.int/iris/bitstream/10665/66856/1/WHO_FCH_ CAH_01.02.pdf. Accessed on 14 Jan 2016.

[2] Morice A. H. and Kastelik. J. A. Cough. 1: Chronic cough in adults. Thorax 2003; 58: 901-7.

[3] Dicpinigaitis PV, Colice GL, Goolsby MJ, Rogg GI, Spector SL, Winther B. Acute cough: a diagnostic and therapeutic challenge. Cough 2009; 5: 11 .

[4] Carter E. R., DebleyJ. S, and Redding G. R. Chronic productive cough in school children: prevalence and associations with asthma and environmental tobacco smoke exposure. Cough 2006; 2: 11.

[5] Worrall G. Acute cough in children. Canadian Family Physician 2011; 57: 315-8.

[6] Asthma Foundation. What is a chronic persistent wet cough? 2010 [cited 201615 Jan 2016]; Available from: http://asthmafoundation.org.nz/wpcontent/uploads/2012/03/Whatis_PersistentWetCough.pdf. Accessed on 14 Jan 2016.

[7] Goldsobel, A. B. and Chipps B. E. Cough in the pediatric population. J Pediatr 2010; 156: 352-8.

[8] Chang, A. B, Oppenheimer J. J, Weinberger M, Rubin B. K, Irwin R. S. Children With Chronic Wet or Productive CoughTreatment and Investigations: A Systematic Review. Chest 2016; 149: 120-42.

[9] Dhar R. Role of mucolytics in wet cough. J Assoc Physicians India 2013; 61: 23-7.

[10] Malerba M. and Ragnoli B. Ambroxol in the 21st century: pharmacological and clinical update. Expert Opin Drug Metab Toxicol 2008; 4: 1119-29.

[11] Ciprandi G, Tosca M, Ricca V, Passalacqua G, Fregonese L, Fasce L, et al. Cetirizine treatment of allergic cough in children with pollen allergy. Allergy 1997; 52: 752-4.

[12] Del Cuvillo A, Sastre J, Montoro J, Jauregui I, Ferrer M, Davila I, et al. Use of antihistamines in pediatrics. JInvestig Allergol Clin Immunol 2007; 17: 28-40.
[13] Ioan I, Poussel M, Coutier L, Plevkova J, Poliacek I, Bolser D.C et alWhat is chronic cough in children? Front Physiol 2014; 5: 322 .

[14] Bailey E. J. and Chang A. In children with prolonged cough, does treatment with antibiotics have a better effect on cough resolution than no treatment?: Part A: Evidence-based answer and summary. Paediatr Child Health 2008; 13: 512-3.

[15] Bell D. S. Combine and conquer: advantages and disadvantages of fixed-dose combination therapy. Diabetes Obes Metab 2013; 15 291-300.

[16] Ibiapina Cda C, Sarinho ES, Camargos PA, Andrade CR, Cruz Filho AA. Allergic rhinitis: epidemiological aspects, diagnosis and treatment. J Bras Pneumol 2008; 34: 230-40.

[17] Demoly P, Calderon MA, Casale T, Scadding G, AnnesiMaesano I, Braun JJ, et al. Assessment of disease control in allergic rhinitis. Clin Transl Allergy 2013; 3: 7.

[18] Stoelzel K, Bothe G, Chong PW and Lenarz M. Safety and efficacy of Nasya/Prevalin in reducing symptoms of allergic rhinitis. Clin Respir J 2014; 8: 382-90.

[19] Benich J. J, Carek P. J. Evaluation of the Patient with Chronic Cough. American Family Physician 2011; 8: 887-92.

[20] Chung K. F. Currently available cough suppressants for chronic cough. Lung 2008; 18: p. S82-7.

[21] Shields M. D, Bush A, Everard M. L, McKenzie S, Primhak R. BTS guidelines: Recommendations for the assessment and management of cough in children. Thorax 2008; 63: iii1-iii15.

[22] Kelley L. K. and AllenP. J. Managing acute cough in children: evidence-based guidelines. Pediatr Nurs 2007; 33: 515-24.

[23] Ryan T, Brewer M, and Small L. Over-the-counter cough and cold medication use in young children. Pediatr Nurs 2008; 34 : 174-80, 184.

[24] Dart R. C, Paul I. M, Bond G. R, Winston D. C, Manoguerra A. S, Palmer R. B, et al. Pediatric fatalities associated with over the counter (nonprescription) cough and cold medications. Ann Emerg Med 2009; 53: 411-7.

[25] Roy V, Malhotra R, Tayal V, Bansal A, Gupta KS. Fixed-dose combinations for cough and common cold in India: an assessment of availability and rationality. Fundam Clin Pharmacol 2011; 25: 258-66. 